\title{
Dynamics of muoniated radical states in phthalocyanines
}

\author{
J. Piroto Duarte*, R.C. Vilão, H.V. Alberto, J.M. Gil, Francisco P.S.C. Gil, \\ A. Weidinger, N. Ayres de Campos \\ Physics Department, University of Coimbra, P-3004 516 Coimbra, Portugal
}

\begin{abstract}
This work reports a spectroscopic and spin-dynamics $\mu \mathrm{SR}$ study of the positive muon states formed in the phthalocyanines $\mathrm{Zn}-\mathrm{Pc}$, $\mathrm{H}_{2}$ $\mathrm{Pc}$ and $\mathrm{Cu}-\mathrm{Pc}$. In $\mathrm{Zn}-\mathrm{Pc}$ and $\mathrm{H}_{2}-\mathrm{Pc}$, three distinct muoniated radicals are formed, seen to undergo spin-dynamical processes with charge carriers. Two of the states have very similar hyperfine interaction values, and are believed to be due to muon addition at the outer benzene rings. The third one has a significantly lower hyperfine constant, and is tentatively assigned to addition at an inner part of the molecule. In $\mathrm{Cu}-\mathrm{Pc}$, the $\mu \mathrm{SR}$ signals found are diamagnetic-like, and the muon seems to be shielded from interactions with charge carriers and local molecular motion.
\end{abstract}

(C) 2005 Elsevier B.V. All rights reserved.

Keywords: Organic semiconductors; Phthalocyanine; Muoniated radicals; Spin dynamics

\section{Introduction}

Research on the subject of organic semiconductors has seen a remarkable increase in recent years, in much due to the growing belief that these materials will be on the very forefront of the next generation of electronic devices. Easy tuning of optical, electrical and even mechanical properties via simple methods such as chemical modification or doping is seen as their major novelty towards the traditional semiconductor [1], even though they still keep large similarities in electronic behaviour with it that promptly permits adapting concepts and procedures wellknown to the scientific and the industrial environments.

Nevertheless, achievements with these new materials are still pending on the somewhat incomplete understanding of charge-carrier transport. Its study is usually addressed using macroscopic measurements such as resistivity or Hall effect [2], but these focus on the slow component of the transport, and albeit providing valuable information, are insufficient to picture microscopic features which are likely to be most significant in the conduction properties of organic semiconductors. Such importance is due to the molecular, hence localised, electronic structure of organic

*Corresponding author. Tel.: +351239410 685; fax: + 351239829158 .

E-mail address: piroto@ci.uc.pt (J. Piroto Duarte). compounds. Local probe techniques as $\mu \mathrm{SR}$ are therefore fundamental to achieve that local picture.

This work reports a $\mu$ SR study on muonium states and dynamics obtained for the organic semiconductor phthalocyanine $(\mathrm{Pc})$, a promising material for applications in solar cells. Three representative types of the Pc family were addressed, differing only on the central inclusion: $\mathrm{Zn}-\mathrm{Pc}$, with a non-magnetic metallic central inclusion, $\mathrm{Cu}-\mathrm{Pc}$, with a magnetic metallic inclusion, and $\mathrm{H}_{2}-\mathrm{Pc}$, with a nonmetallic inclusion (also of non-magnetic character). We present spectroscopic results about the states formed in these compounds, and discuss the origin of the dynamical signals observed in longitudinal field geometry.

\section{Experimental details and results}

Experimental measurements were performed on $\beta$-form, undoped powder samples with the GPS and DOLLY spectrometers at PSI (high TF geometry) and the EMU spectrometer at ISIS (LF geometry) in a broad temperature range $(5-600 \mathrm{~K})$. Transverse geometry measurements at high fields aimed at identifying and characterising the muon states, while longitudinal fields were used to investigate the dynamics of the states. 


\subsection{Transverse-field measurements}

Fig. 1 shows a high-statistics Fourier transform $\mu \mathrm{SR}$ spectrum of Zn-Pc obtained at PSI under $0.4 \mathrm{~T}$ at $500 \mathrm{~K}$. Three distinct pairs of lines are observed, corresponding to the formation of three muoniated radicals. Two of those had already been identified (states I and II), and their isotropic hyperfine interactions determined to be $A_{\mathrm{I}}=$ $127 \mathrm{MHz}$ and $A_{\text {II }}=142 \mathrm{MHz}$ [3]. The new state, which we label state III, has a much lower hyperfine interaction of $A_{\text {III }}=20(2) \mathrm{MHz}$. An additional broad component under the diamagnetic line suggests also the presence of weak paramagnetic interactions. Time-domain fits reveal that the two high-hyperfine interaction states have a formation probability of about $14 \%$ each, while state III and the broad component exhibits a population of around $25 \%$ each. Within errors, and accounting for the small diamagnetic contribution, total population values are consistent with no missing fraction.

Similar results were found for $\mathrm{H}_{2}-\mathrm{Pc}$, although both the populations and the hyperfine interactions of states I and II are slightly lower. Since those two states were also harder to resolve, time-domain fits were carried out using only one component to describe them; the average hyperfine interaction of states I and II was found to be $A_{\mathrm{I} / \mathrm{II}}=112(1) \mathrm{MHz}$. State III was seen to be the dominant state, with a population of about $80 \%$, in opposition to the $5 \%$ obtained for both the broad component and the set of states I/II. The fitted hyperfine interaction of state III is $A_{\text {III }}=26(3) \mathrm{MHz}$, slightly larger than what happens in $\mathrm{Zn}-\mathrm{Pc}$.

Temperature-dependent measurements taken with $\mathrm{Zn}-\mathrm{Pc}$ show the shift of the hyperfine interaction of states I and II to lower values with increasing temperature, as depicted in Fig. 2. This shift was seen to be consistent with a

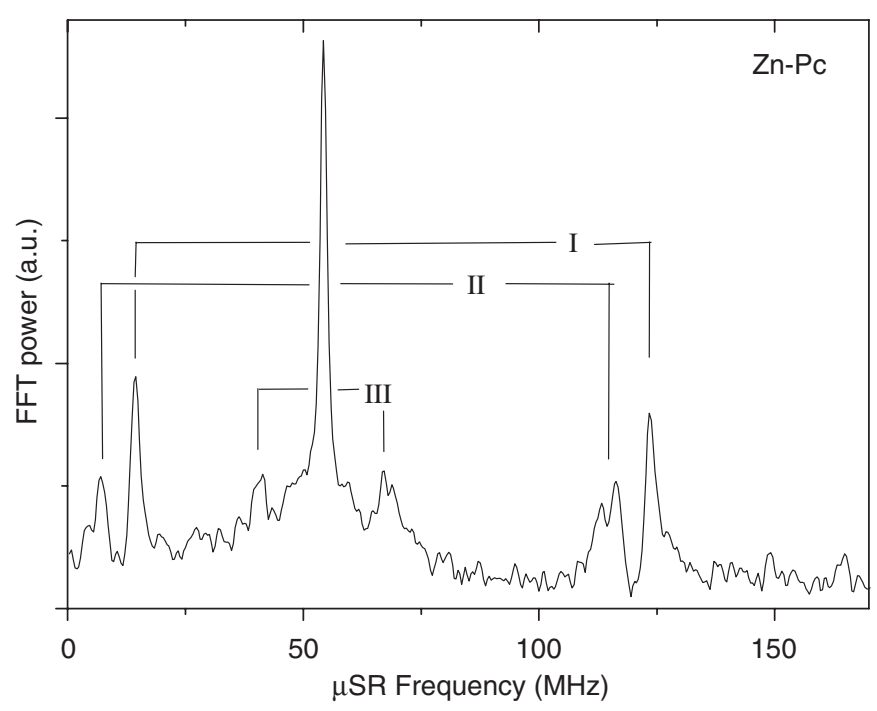

Fig. 1. Fourier power spectrum of $\mathrm{Zn}-\mathrm{Pc}$ in a transverse field of $0.4 \mathrm{~T}$ at $500 \mathrm{~K}$. The three pairs of lines identified by the Roman numerals I-III indicate the formation of at least three distinct muoniated radical states in this compound.

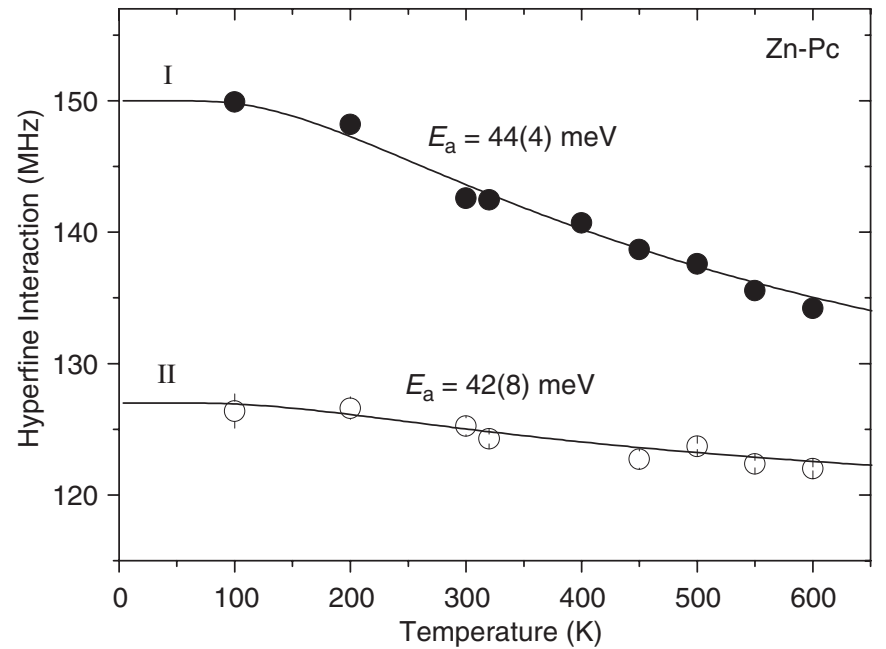

Fig. 2. Temperature dependence of the hyperfine interaction of states I and II in $\mathrm{Zn}-\mathrm{Pc}$. The solid lines indicate a temperature activated fit with the law $A(T)=A_{0}+\left(A_{\infty}-A_{0}\right) \exp \left(-E_{a} / k T\right)$.

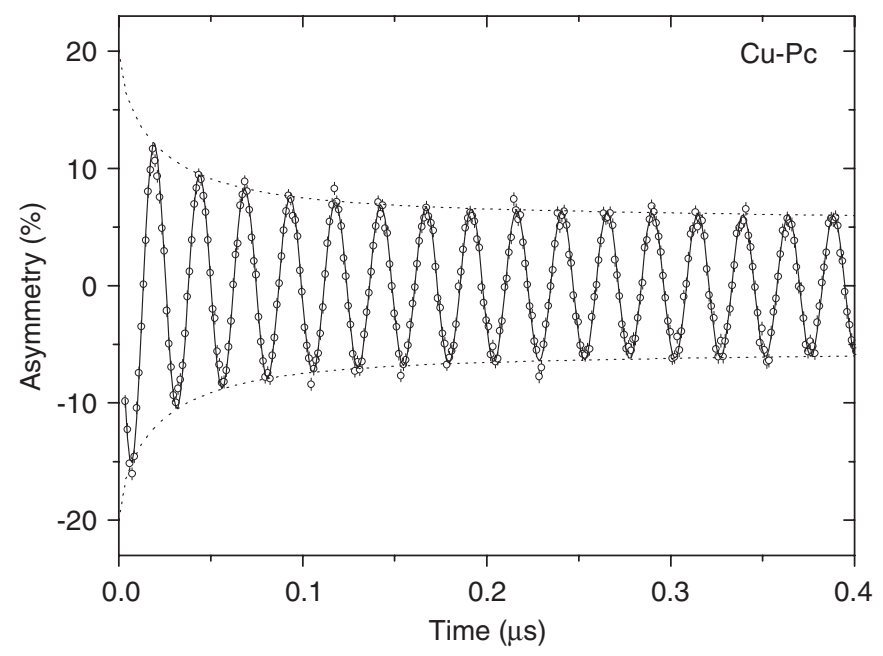

Fig. 3. $\mu$ SR time spectrum of Cu-Pc in a transverse field of $0.3 \mathrm{~T}$ at $200 \mathrm{~K}$. The solid line is the corresponding fit as described in the text; the dashed lines depict the fit function's envelope.

temperature-activated process of activation energy around $40 \mathrm{meV}$.

$\mathrm{Cu}-\mathrm{Pc}$ exhibits two distinct diamagnetic-like signals with drastically different depolarisation rates (Fig. 3). The fast relaxing component is well fitted with a Lorentziandamped precession, whose depolarisation rate of around $10 \mu \mathrm{s}^{-1}$ is almost three orders of magnitude larger than the slow relaxing component's one. Both depolarisation rates exhibit a very weak temperature dependence.

\subsection{Longitudinal-field measurements}

For $\mathrm{Zn}-\mathrm{Pc}$ and $\mathrm{H}_{2}-\mathrm{Pc}$, longitudinal field time measurements were taken as a function of temperature. The spectra obtained allow no distinction of the four signal components seen in transverse field, but the data are well fitted with a simple Lorentzian function. In $\mathrm{Zn}-\mathrm{Pc}$, the relaxation 


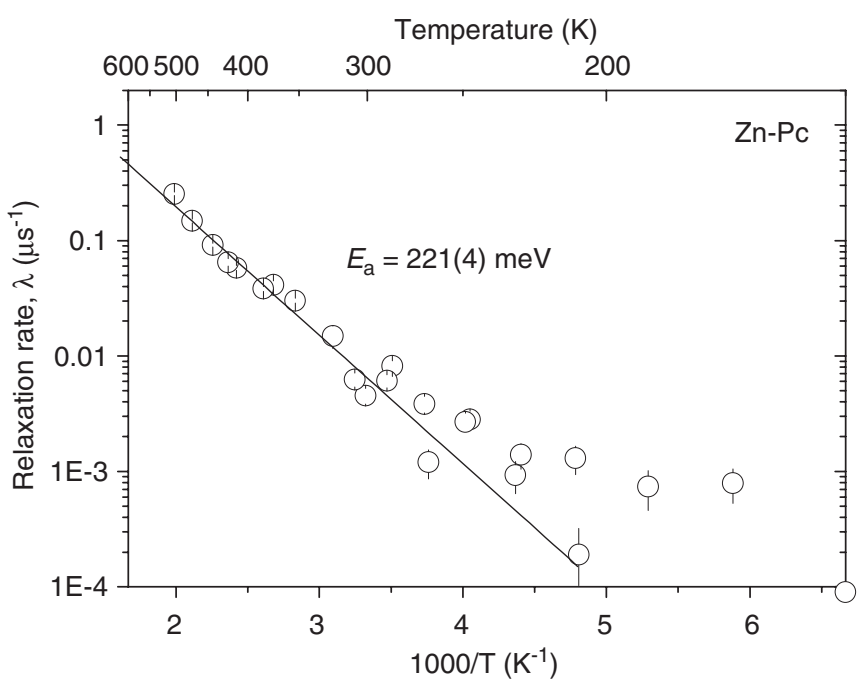

Fig. 4. Arrhenius plot of the relaxation rate observed in undoped $\mathrm{Zn}-\mathrm{Pc}$ in a longitudinal field of $0.1 \mathrm{~T}$.

rate starts increasing at around $200 \mathrm{~K}$, following a temperature-activated law of activation energy $221 \mathrm{meV}$ (Fig. 4). Similar findings are seen in $\mathrm{H}_{2}-\mathrm{Pc}$, although the collected data is insufficient to quote an activation energy value.

In $\mathrm{Cu}-\mathrm{Pc}$, the two components identified in transverse field are again observed in longitudinal field. Likewise, the Lorentzian relaxation rates are almost temperature independent, differ in more than two orders of magnitude and have absolute values equal within error to the transverse field depolarisation rates.

\section{Discussion}

\subsection{Radical states: $\mathrm{Zn}-\mathrm{Pc}, \mathrm{H}_{2}-\mathrm{Pc}$}

Following the general understanding of how muoniated radicals are formed in unsaturated compounds, the three radical states observed in $\mathrm{Zn}-\mathrm{Pc}$ and $\mathrm{H}_{2}-\mathrm{Pc}$ must be due to muonium addition at a molecule's double bond. The similar hyperfine interactions of states I and II suggest very similar sites for the muon in those states; the strongest candidates seem to be the two carbons at the ortho and meta positions in the outer benzene rings [3]. State III, on the other hand, must be located at a quite different site. Anchoring at the bridging nitrogen seems the most reasonable option, since except for the outer rings, it is the region less populated by atoms in the molecule's plane. Off-plane positions would be unlikely due to the molecular stacking of phthalocyanines.

In organic compounds, the origin of $\mu \mathrm{SR}$ spin dynamics may arise either from molecular motion or from scattering with charge carriers. The first is more usual in insulating materials [4], whereas the second is observed in conducting ones [5]. In the phthalocyanine's case, it is due to spincattering with charge-carriers, since the activation energy of the LF relaxation rate was found to be quite different from the activation energy of the hyperfine interaction shift. This means that the phonon modes responsible for local molecular motion, which couple to the hyperfine frequency and shift it to lower values, are not the same as those that couple to the process responsible for the LF spin dynamics. Therefore, LF spin dynamics may only be attributed to charge-diffusion processes.

\subsection{Diamagnetic states: $\mathrm{Cu}-\mathrm{Pc}$}

The diamagnetic signals found in $\mathrm{Cu}-\mathrm{Pc}$ are due to the magnetic character of this molecule, since the unpaired electron from the $\mathrm{Cu}$ atom spin pairs with the muonium's electron. This happens at all possible muonium addition sites, which we assume to be the same as those in $\mathrm{Zn}-\mathrm{Pc}$ and $\mathrm{H}_{2}-\mathrm{Pc}$. The fact that there are two distinct signals, one fast relaxing and the other slowly relaxing, may have to do with the quantum-mechanical details of this two-spin coupling.

Contrary to what happens with $\mathrm{Zn}-\mathrm{Pc}$ and $\mathrm{H}_{2}-\mathrm{Pc}$, the TF and $\mathrm{LF}$ relaxation rates observed in $\mathrm{Cu}-\mathrm{Pc}$ are not due to scattering with charge carriers, nor to local molecular dynamics, as their temperature dependence is very weak. The small depolarisation rate values of the slow component have the right order of magnitude to be attributed to the dipolar interaction of the muon with nearby protons, while the high values of the fast component seem to indicate the involvement of electronic spins in its origin.

\section{Conclusion}

Spectroscopic and spin-dynamics $\mu \mathrm{SR}$ results were presented for the $\mathrm{Zn}-\mathrm{Pc}, \mathrm{H}_{2}-\mathrm{Pc}$ and $\mathrm{Cu}-\mathrm{Pc}$ phthalocyanines. Three distinct muoniated radicals were identified in Zn-Pc; two of them, with slightly different hyperfine interactions, were attributed to addition at the outer benzene rings, while the third one was tentatively assigned to addition at a bridging nitrogen. In $\mathrm{H}_{2}-\mathrm{Pc}$ similar states were found, but the exact hyperfine parameters and populations are different. The observed longitudinal field relaxation was attributed to charge-carrier spin scattering. In $\mathrm{Cu}-\mathrm{Pc}$, only diamagnetic signals were observed, but it is assumed that the same or similar sites are occupied as in $\mathrm{Zn}-\mathrm{Pc}$. The LF and TF relaxations were found not to be due to charge-carrier scattering nor to molecular motion.

\section{Acknowledgments}

The assistance of all LMU staff at PSI and $\mu$ SR instrument scientists at ISIS is gratefully acknowledged. This work was performed at the Swiss Muon Source, Paul Scherrer Institute, Villigen, Switzerland, and partially supported by the European Commission under the 6th Framework Programme through the Key Action: Strengthening the European Research Area, Research Infrastructures, contracts no: RII3-CT-2004-505925 and no: HII3CT-2003-505925. We also thank the support of the 
Portuguese Foundation for Science and Technology (FCT) and the PRODEP III program under action 5.3.

\section{References}

[1] A.J. Heeger, Rev. Mod. Phys. 73 (2001) 681.
[2] M. Pfeiffer, et al., Appl. Phys. Lett. 73 (1998) 3202.

[3] J. Piroto Duarte, et al., Physica B 326 (2003) 94.

[4] F.L. Pratt, et al., Magn. Reson. Chem. 38 (2000) S27.

[5] F.L. Pratt, et al., Phys. Rev. Lett. 79 (1997) 2855. 